\title{
Satisfacción con la vida en escolares de la ciudad de Arequipa
}

\section{Satisfaction with Life in High School Students from Arequipa}

\author{
Walter Lizandro Arias Gallegos* ${ }^{*}$ Universidad Católica San Pablo, Arequipa, \\ Perú. ORCID: https://orcid.org/0000-0002-4183-5093
}

Julio Cesar Huamani Cahua (1) Universidad Católica San Pablo, Arequipa, Perú. ORCID: https://orcid.org/0000-0001-8159-803X

Tomás Caycho-Rodríguez@ Universidad Privada del Norte, Lima, Perú. ORCID: https://orcid.org/0000-0002-5349-7570

Recibido 05-01-18 Revisado 15-01-18 Aprobado 26-02-18 En línea 27-02-18

*Correspondencia

Email: walterlizandro@hotmail.com

\section{Citar como:}

Arias W., Huamani, J., \& Caycho-Rodríguez, T. (2018). Satisfacción con la vida en escolares de la ciudad de Arequipa. Propósitos y Representaciones, 6(1), 351-407. doi: http://dx.doi. org/10.20511/pyr2018.v6n1.206

(C) Universidad San Ignacio de Loyola, Vicerrectorado de Investigación, 2018

(cc) BY-NC-ND Este artículo se distribuye bajo licencia CC BY-NC-ND 4.0 Internacional (http://creativecommons.org/licenses/by-nc-nd/4.0/). 


\section{Resumen}

El presente estudio, que se enmarca dentro de la Psicología Positiva Educativa, tiene dos objetivos, analizar psicométricamente la Escala de Satisfacción con la Vida, y comparar los valores resultantes en función del sexo, la edad y la institución de procedencia, en un grupo de escolares de la ciudad de Arequipa. Se trabajó con una muestra de 872 estudiantes de 4to y 5 to de media de cinco escuelas de gestión mixta y diferenciada, donde el $46.9 \%$ son varones y el $53.1 \%$ mujeres, obtenida por métodos probabilísticos. Se utilizó la Escala de Satisfacción con la Vida de Diener. Los resultados indican que la prueba cuenta con una estructura factorial unidimensional y una confiabilidad adecuada para variables latentes, $(\omega=.99$ y $\mathrm{H}=.99)$. Las comparaciones efectuadas sugieren que los varones y los estudiantes de mayor edad presentan niveles mayores de satisfacción con la vida.

Palabras clave: Satisfacción con la vida, psicología positiva, educación, psicometría.

\section{Summary}

This study, in the framework of the Positive Educational Psychology, has two objectives: to perform a psychometric analysis of the Satisfaction with Life Scale, and to compare the resulting values according to gender, age and educational institution, in a group of schoolers from the city of Arequipa. We worked with a sample of 872 students in the 4th and 5th year of high school, from five mixed and single-sex schools. Besides, $46.9 \%$ of this sample are male students and $53.1 \%$ are female students. The sample was obtained by probabilistic methods. The Diener's Satisfaction with Life Scale was used. The outcomes indicate that the test has a one-dimensional factorial structure and suitable reliability for latent variables $(\omega=.99$ and $\mathrm{H}=.99)$. The comparisons suggest that, male students and those who are older have higher levels of satisfaction with life. 
Keywords: Satisfaction with life, Positive Psychology, Education, Psychometry. 


\section{Introducción}

La investigación contemporánea señala que el bienestar subjetivo (SWB, por sus siglas en inglés) es un constructo importante y relevante para la psicología (Wu \& Yao, 2006) que tiene tres componentes: el afecto positivo, afecto negativo y satisfacción con la vida (SWL, por sus siglas en inglés) (Pavot \& Diener, 2008). Este último, la SWL, es la dimensión cognitiva del SWB (Diener, Emmons, Larsen \& Griffin, 1985; Diener, Oishi \& Lucas, 2003) y es definida como el grado en que una persona evalúa la calidad global de su vida en conjunto o acerca de dominios específicos como el trabajo, la familia, los amigos, entre otros, sobre la base de sus propios criterios (Diener, 2000; Diener \& Ryan, 2009; Pavot \& Diener, 1993; Proctor, Linley \& Maltby, 2009a; Rodgers, Neville \& La Grow, 2017). Junto con los factores internos, las condiciones externas, como la calidad de vida familiar y la escolaridad, son importantes al momento de realizar evaluaciones globales de la SWL (Suldo \& Shaunessy-Dedrick, 2013).

La SWL es importante para personas de todas las edades, debido a las correlaciones con un conjunto de indicadores de salud mental (Silva, do Céu Taveira, Marques \& Gouveia, 2015). Los estudios acerca de la SWL se han centrado en su mayoría en personas adultas (Erdogan, Bauer, Truxillo \& Mansfield, 2012; Pavot \& Diener 2008), siendo aún pocas las investigaciones en población infantil o adolescente, al menos en los países de América Latina (Castro, 2012; Arias, Espiñeira \& Huamani, 2017). Sin embargo, en las últimas décadas se observa un aumento de las investigaciones centradas en niños y adolescentes (Gilman \& Huebner 2006; Goldbeck, Schmitz, Besier, Herschbach \& Henrich, 2007; Pavot \& Diener, 2008; Proctor, Linley \& Maltby, 2009b).

Así, el estudio de la SWL en adolescentes es un componente clave de las evaluaciones integrales del SWB de los adolescentes debido a sus implicancias para el buen funcionamiento psicológico, social, educativo y físico (Greenspoon \& Saklofske, 2001; Moksnes, Løhre, Byrne \& Haugan, 
2014; Proctor et al., 2009a; Suldo, Huebner, Friedrich \& Gilman, 2009). La satisfacción con la vida se ha estudiado en contextos educativos, donde, adolescentes en edad escolar con altos niveles de SWL y psicopatología mínima presentan mejores relaciones sociales, logros educativos y salud física, que aquellos con psicopatología mínima pero con bajos niveles de SWL (Suldo \& Shaffer, 2008). Asimismo, adolescentes escolares con una alta SWL evidencian actitudes más favorables hacia los docentes y la escuela (Gilman \& Huebner 2006), así como un mayor compromiso académico (Lewis, Huebner, Malone \& Valois, 2011) y aspiraciones académicas (Proctor, Linley \& Maltby, 2010). Por otro lado, los adolescentes con mayor SWL, favorecidos por un clima familiar positivo (Luna, Laca, \& Mejía, 2011), tienen menor riesgo de ser víctimas de acoso (Povedano, Hendry, Ramos \& Varela, 2011; Totan, Özer \& Özmen, 2017). Otro estudio, señala que las valoraciones positivas que hace el profesor del alumno se relacionan con niveles más altos de SWL (Martínez-Antón, Buelga \& Cava, 2007). También se han establecido relaciones entre la SWL y las metas académicas (Díaz, \& Martínez, 2004), un mejor aprovechamiento del tiempo libre (Pavot \& Diener, 1993), auto evaluaciones nucleares positivas (Tan, Yang, Ma \& Yu, 2016), menor vulnerabilidad a las consecuencias del desempleo familiar (Frasquilho, Matos, Neville, Gaspar \& Almeida, 2016) y menos probabilidad de ocurrencia de comportamientos riesgosos (Çakar, Tagay \& Karataş, 2015). La SWL se relaciona con la resiliencia en estudiantes de nivel secundario (Salgado, 2009), cuyo efecto positivo combinado influye en la posibilidad de culminación de los estudios (Bernal, Daza \& Jaramillo, 2015).

Teniendo en consideración que la SWL es diferente en la adolescencia, en comparación a otras etapas de desarrollo, debido a los múltiples cambios biológicos, psicológicos, sociales y cognitivos que ocurren durante este periodo (Goldbeck et al., 2007), en los últimos años, mucho de la investigación en adolescentes ha tenido como objetivo examinar los determinantes del SWB, destacando la importancia de identificar diversos factores que promueven la SWL (Proctor et al., 2009b). Uno de estos factores es la edad. Los resultados 
de un estudio transnacional (Woynarowska, Tabak \& Mazur, 2004), realizado en diversos países europeos, indicó una disminución de la SWL con la edad, lo que es similar a lo reportado en adolescentes australianos entre los 12 y 16 años (Meuleners, Lee, Binns \& Lower, 2003). Contrario a estos resultados, una investigación realizada a gran escala en 63 escuelas públicas de Carolina del Sur, en Estados Unidos (Huebner, Suldo, Valois, Drane \& Zullig, 2004), reportó la ausencia de cambios en la SWL de los estudiantes de 9no a $12 \mathrm{mo}$ grado. En el Perú, por otro lado, se han reportado diferencias significativas de la satisfacción con la vida en función de la edad (Martínez, 2004, 2006).

En relación al sexo, los estudios con adolescentes, revelan que los hallazgos no han sido concluyentes (Chui \& Wong, 2016). Algunos estudios señalan que los niveles promedio de SWL son muchas veces similares entre adolescentes hombres y mujeres (Casas et al., 2007; Froh, Yurkewicz \& Kashdan, 2009; Gilman \& Huebner, 2006; Huebner, Drane \& Valois, 2000; Huebner et al., 2004). Contrario a esto, otras investigaciones con adolescentes en edad escolar de Estados Unidos (Suldo, Minch \& Hearon, 2015), Alemania (Goldbeck et al., 2007), Perú (Tarazona, 2005), Portugal (Neto, 1993), Turquía (Verkuyten, 1986) y Noruega (Moksnes \& Espnes, 2013) reportan que los hombres presentan niveles de SWL ligeramente (aunque significativos) más altos que las mujeres. Se han analizado también las diferencias de la SWL en función de diferentes niveles socioeconómicos y razas (ver Gilman \& Huebner 2003).

Con el objetivo de evaluar la satisfacción con la vida, se diseñó la Satisfacción with Life Scale (SWLS, Diener et al., 1985), como una medida breve de cinco ítems, destinada a medir el grado de satisfacción de un individuo con su vida que es uno de los instrumentos más utilizados para la medida de la SWL (Silva et al., 2015; Oishi, 2006) y que tiene mucha utilidad para fines de investigación (Pavot \& Diener 1993; 2008). Los cinco ítems están codificados en dirección positiva, por lo que pueden sumarse para lograr un puntaje total para la escala (Diener et al., 1985). 
La SWLS se ha utilizado en más de 4000 estudios (Gouveia, Milfont, Da Fonseca \& de Miranda Coelho, 2009), siendo traducida a numerosos idiomas como el chino (Sachs, 2004), francés (Fouquereau \& Rioux 2002), turco (Durak, Senol-Durak \& Gencoz, 2010), noruego (Vittersø, Biswas-Diener \& Diener, 2005), sueco (Hultell, \& Gustavsson, 2008), taiwanés (Wu \& Yao, 2006), español (Atienza, Pons, Balaguer \& García-Merita, 2000; Pons, Atienza, Balaguer \& García-Merita, 2002), koreano (Lim, 2015), entre otros; además de ser utilizado en diversos grupos como adultos mayores (Pons et al., 2002), niños (Atienza et al., 2000), pacientes ambulatorios no psiquiátricos (Arrindell, Meeuwesen \& Huyse, 1991), mujeres embarazadas (Cabañero et al., 2004) y otros. Sin embargo, debido a la creciente necesidad de estudios sobre la SWL en adolescentes, que se ve evidenciado por los numerosos estudios a nivel internacional (Atienza, Balaguer \& García-Merita, 2003; Gilman \& Huebner 2001; Neto, 1993), se realizó la traducción al español y validación de la SWLS en esta población (Atienza et al., 2000; Pons et al., 2002).

Respecto a las propiedades psicométricas de la SWLS, una investigación que analizó seis estudios, indicó que los valores del coeficiente alfa para la SWLS varían entre .79 a .89, indicando una alta consistencia interna (Pavot \& Diener, 1993). Un metanálisis más reciente (Vassar, 2008), que incluyó datos de confiabilidad de 62 estudios que utilizan la SWLS, reportó que el valor promedio del coeficiente alfa de Cronbach fue de .78 con un intervalo de confianza al 95\% (IC95\%) de .766 a .807 y donde la versiones en inglés y las muestras de jóvenes demostraron tener una mejor confiabilidad de las puntuaciones. Estos hallazgos coinciden con otros resultados que presentan coeficientes alfa de Cronbach que varían entre .70 y .90 (Gouveia et al., 2009; Laranjeira 2009; Neto, 1993; 2001; Pavot, Diener, Colvin \& Sandvik, 1991; Simões, 1992) y otros superiores a .90 (Lent, do Céu Taveira, Sheu \& Singley, 2009; Sancho, Galiana, Gutierrez, Francisco, \& Tomás, 2014). En base a estos resultados, se puede considerar la existencia de un alto grado de variabilidad entre diferentes poblaciones (Pavot \& Diener 2008). 
En relación a estructura factorial de la SWLS, el estudio original (Diener et al., 1985), realizó un análisis de ejes principales resultando una sola dimensión cognitiva. A pesar de que el análisis de la estructura factorial de la SWLS es menos estudiada en adolescentes (Atienza et al., 2000), investigaciones realizadas con otras muestras replican la estructura de factor único (Atienza et al., 2003; Gouveia et al., 2009; Lewis, Shevlin, Smekal \& Dorahy, 1999; Neto 1993; Oladipo \& Balogun 2012; Pavot \& Diener, 1993; Pons et al., 2002; Sancho et al., 2014). Sin embargo, un estudio con estudiantes universitarios suecos (Hultell \& Gustavsson, 2008) reportó que un modelo de segundo orden de dos factores presentó índices de ajuste superior en comparación con el modelo unidimensional; mientras que otra investigación con estudiantes universitarios de Taiwán (Wu \& Yao, 2006) señaló que tanto el modelo de factor único y el de dos factores presentaron un ajuste aceptable. Estos resultados sugieren que la estructura interna de la SWLS puede ser reconsiderada (Hultell \& Gustavsson, 2008). Aunque diversos estudios han evaluado aspectos psicométricos de la SWLS, una revisión de la literatura indica la ausencia de este tipo de investigaciones en adolescentes peruanos.

En el Perú, la SWLS ha sido utilizada en diversos estudios (Alarcón, 2000, 2001; Salgado, 2006; Martínez, 2004, 2006; Velásquez et al., 2016), pero solo unos pocos han estado orientados a la población adolescente (Salgado, 2009; Tarazona, 2005). Todos estos estudios, han tenido lugar en la ciudad de Lima, donde Alarcón (2000) realizó la primera valoración psicométrica de la SWLS, en estudiantes universitarios, reportando adecuados valores de validez, pero bajos índices de confiabilidad. En Arequipa, por otro lado, no hay investigaciones sobre la SWL, ya que no se cuenta con instrumentos debidamente validados, para la población adolescente de la región. Sin embargo, sí existen reportes que dan cuenta de una realidad preocupante para los adolescentes de la región, donde se evidencia un muy bajo sentido de vida, lo que supone la ausencia de un proyecto futuro bien establecido, el 
riesgo muy alto de padecer neurosis (Huamani \& Ccori, 2016) y una mayor presencia de sintomatología depresiva (Rivera, Arias \& Cahuana, 2017).

En este contexto, el desarrollo de las emociones positivas en la niñez y adolescencia, convierte a la escuela en un espacio privilegiado para su promoción y su intervención (Oros, Manucci \& Richaud, 2011). Desde la Psicología Positiva, se han realizado diversas aplicaciones en el campo educacional que han buscado fomentar un mayor bienestar psicológico y satisfacción vital a través de la experimentación de emociones positivas y el diseño de entornos educativos flexibles (Adler, 2017; Bisquerra \& Hernández, 2017; Merchán, Bermejo \& González, 2014; Palomera, 2017). Para una medida adecuada del impacto de estas iniciativas se hace necesario contar con instrumentos de medida de la SWL validados para adolescentes (Alfaro et al., 2016).

Por lo anteriormente mencionado, el objetivo del presente estudio es doble. Por un lado, analizar las evidencias de validez de constructo y fiabilidad de la SWLS para su empleo en estudiantes de nivel secundario de la ciudad de Arequipa y, en segundo lugar, analizar la satisfacción con la vida de estos estudiantes en función del sexo, la edad y la institución educativa de la que proceden.

\section{Método}

El presente estudio es por una parte de tipo instrumental y por otra de tipo asociativo (Ato, López \& Benavente, 2013; Montero \& León, 2007), en función de los objetivos de investigación. Siguiendo la tipología de Hernández, Fernández y Baptista (1997) se trata de un diseño de investigación no experimental, descriptivo-transversal. 


\section{Participantes.}

La población estuvo conformada por estudiantes del cuarto y quinto de secundaria de cinco instituciones educativas públicas de la ciudad de Arequipa ( 2 de varones, 2 de mujeres y 1 mixta), que se presentan en la Tabla 1.

\section{Tabla 1.}

Distribución de frecuencias y porcentajes de la población de estudiantes del cuarto y quinto de secundaria

\begin{tabular}{|c|c|c|c|c|c|c|}
\hline \multirow{3}{*}{ Instituciones Educativas } & \multicolumn{4}{|c|}{ Grado de estudios } & \multirow{2}{*}{\multicolumn{2}{|c|}{ Total }} \\
\hline & \multicolumn{2}{|c|}{ 4to } & \multicolumn{2}{|r|}{ 5to } & & \\
\hline & fi & $\%$ & fi & $\%$ & fi & $\%$ \\
\hline Institución Educativa 1 & 195 & 25.90 & 182 & 24.80 & 377 & 25.35 \\
\hline Institución Educativa 2 & 110 & 14.61 & 108 & 14.71 & 218 & 14.66 \\
\hline Institución Educativa 3 & 198 & 26.29 & 208 & 28.34 & 406 & 27.30 \\
\hline Institución Educativa 4 & 113 & 15.01 & 110 & 14.99 & 223 & 15.00 \\
\hline Institución Educativa 5 & 137 & 18.19 & 126 & 17.17 & 263 & 17.69 \\
\hline Total & 753 & 100.00 & 734 & 100.00 & 1487 & 100.00 \\
\hline
\end{tabular}

La muestra se seleccionó de manera probabilística mediante el método de muestreo estratificado y quedó constituida por 872 estudiantes de 4to y 5to año se secundaria procedentes de cinco instituciones educativas de Arequipa, con una edad promedio de 15 años (D.E. $=0.8$ ), dentro de un rango de 14 a 18 años. El 46.9\% son varones y el 53.1\% mujeres. El 51.8\% fueron del 4 to de secundaria y $48.2 \%$ del 5 to de secundaria.

\section{Instrumentos.}

Se aplicó la Escala de Satisfacción con la Vida (SWLS; Diener et al., 1985), que fue adaptada al idioma español por Atienza et al., (2000), y validada para el contexto peruano por Alarcón (2000). La prueba consta de cinco ítems en una escala de respuesta tipo Likert con cinco alternativas de respuesta ("totalmente en desacuerdo $=1$ " a "totalmente de acuerdo $=5$ "), donde 
puntuaciones más altas indican un mayor grado de SWL. La prueba puede ser aplicada desde los 11 años y tiene una duración promedio de 3 minutos (Alarcón, 2000).

\section{Procedimiento.}

En primer lugar, se procedió a solicitar los permisos ante los directores de las instituciones educativas escogidas. Una vez obtenidos estos, se procedió a hacer las coordinaciones respectivas para fijar las fechas de evaluación y los procedimientos a seguir. El recojo de la data se llevó a cabo en horas de clase, con el permiso del profesor. A los estudiantes, se les explicó los fines del estudio y se recalcó la confidencialidad de sus datos. Todos los estudiantes participaron voluntariamente y firmaron un consentimiento informado. Los datos fueron recogidos entre los meses de agosto y octubre del 2016.

\section{Análisis de datos.}

La confiabilidad de las variables latentes se estimó mediante el cálculo del coeficiente Omega ( $\omega$; Mcdonald, 1999; Ventura-León \& Caycho-Rodríguez, 2017) y coeficiente H (Hancock \& Mueller, 2001) cuyos valores > 80 indican adecuada confiabilidad (Raykov \& Hancock, 2005).

En relación a la evidencia de validez de constructo, se verificó la estructura interna mediante el Análisis Factorial Confirmatorio (AFC). No se efectuó un Análisis Factorial Exploratorio (AFE) porque teóricamente la escala asume la unidimensionalidad. El AFC se realizó teniendo como base la matriz de correlaciones policóricas y la estimación de máxima verosimilitud (Hair, Anderson, Tatham \& Black, 2014). Se calculó el valor de la chi-cuadrado relativa ( $\chi 2$ /df; Bollen, 1998), cuyos valores $\leq 3$ expresan un ajuste adecuado (Carmines \& McIver, 1981). Teniendo en consideración la recomendación de Hu y Bentler (1999) se calcularon el error cuadrático medio de aproximación (RMSEA), el residuo cuadrático medio estandarizado (SRMR), el índice de bondad de ajuste (GFI) y el índice de ajuste comparativo (CFI). Valores de 
GFI y CFI > .90, RMSEA <.08 (Hu \& Bentler, 1999) indican un buen ajuste. Se consideraron que valores de las cargas factoriales estandarizadas $(\lambda) \geq .50$ son adecuados (Johnson \& Stevens, 2001). Se calculó la varianza promedio extraída del factor (AVE; Average Variance Extracted), donde valores $>.50$ brindan evidencia de validez interna convergente (Fornell \& Larcker, 1981; Wixom \& Todd, 2005).

Finalmente, para realizar el tratamiento estadístico de las comparaciones, se aplicó la prueba t de Student y el análisis de varianza. Asimismo, se calcularon los valores del coeficiente $d$ de Cohen (Cohen, 1998) y omega cuadrado ( $\omega^{2}$, Caycho-Rodríguez, 2017; Fritz, Morris \& Richler, 2012) como medidas del tamaño del efecto (TE) de la diferencia entre dos grupos y más de dos grupos independientes respectivamente. Para la interpretación de la $d$, valores de $.20, .50$ y .80 expresan un TE pequeño, moderado y grande; respectivamente (Cohen, 1998; Ferguson, 2009). En el caso del $\omega^{2}$, valores de $0.04,0.25$ y 0.64 indican un TE pequeño, moderado y fuerte, respectivamente (Fritz et al., 2012).

\section{Resultados}

\section{Validez de Constructo y confiabilidad de la SWLS.}

Para realizar el AFC se cargaron los 5 ítems de la SWLS, en una sola variable latente (Figura 1). Los índices de bondad de ajuste indican que el modelo unidimensional ajusta adecuadamente con los datos $(\chi 2 / \mathrm{df}=.281$; GFI $=$ .999; $\mathrm{CFI}=1.00 ; \mathrm{RMSEA}=0.000$ [IC90\% .000, .038]). Las $\lambda$ para el modelo unidimensional fueron significativas y en la dirección esperada (ítem $1=$ .55 ; ítem $2=.96$; ítem $3=1.26$; ítem $4=.99$; ítem $5=.98$ ) con una $\lambda_{\text {promedio }}$ de .948 mayor al .70 recomendado (Hair et al., 2014). Todos los ítems presentan $\lambda$ altas, siendo el ítem tres (Estoy satisfecho(a) con mi vida), el que mejor explica el modelo. Las magnitudes de las $\lambda$ se observan en la Figura 1. El 
valor de AVE fue de .520 , superior a .50 que permite mostrar evidencia empírica de validez interna convergente (Fornell \& Larcker, 1981).

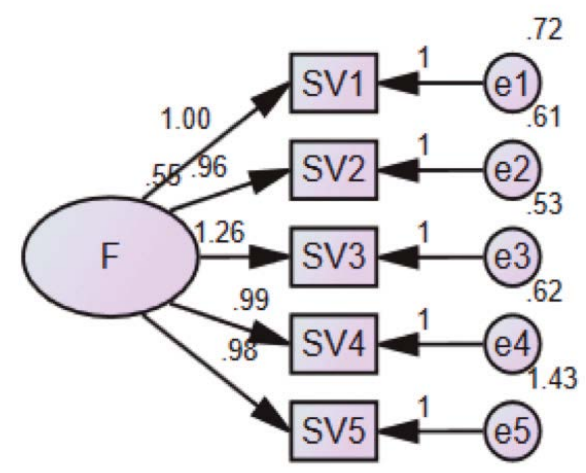

Figura 1. Modelo final estimado de la SWLS

La confiabilidad para variables latentes, calculada mediante los valores del coeficiente $\omega=.99$ y $\mathrm{H}=.99$, indican una confiabilidad adecuada del modelo unidimensional de la SWFLS.

\section{Comparación de la SWL según sexo, edad e institución educativa.}

La Tabla 2 muestra las diferencias de la SWL según sexo. Así pues, al realizar la evaluación global de la vida actual, son los varones quienes perciben de modo más positivo sus circunstancias actuales de forma global en comparación que las mujeres $\left(t_{(870)}=2.048, p=.041, d=.138\right.$ IC95\% .004 - .271). A pesar de la existencia de una diferencia estadísticamente significativa, estos resultados no presentan importancia práctica en base al cálculo del TE $(d<.20)$. En este sentido, los resultados indican que el pertenecer a uno $u$ otro sexo no generaría una diferencia importante en la SWL. 
Tabla 2.

Satisfacción con la Vida según sexo.

\begin{tabular}{|c|c|c|c|c|c|c|c|c|c|}
\hline & \multirow[t]{2}{*}{ Sexo } & \multirow[t]{2}{*}{$\mathrm{N}$} & \multirow[t]{2}{*}{ Media } & \multirow[t]{2}{*}{$\begin{array}{l}\text { Desviación } \\
\text { estándar }\end{array}$} & \multicolumn{2}{|c|}{ Prueba de Levene } & \multicolumn{3}{|c|}{$\begin{array}{l}\text { Prueba } \mathrm{T} \text { para muestras } \\
\text { independientes }\end{array}$} \\
\hline & & & & & $\mathrm{F}$ & $\mathrm{p}$ & $t$ & $g l$ & $p$ \\
\hline \multirow{2}{*}{$\begin{array}{l}\text { Satisfacción } \\
\text { con la Vida } \\
\text { (SWLS) }\end{array}$} & Varón & 409 & 18.99 & 4.07 & \multirow[t]{2}{*}{18.850} & \multirow[t]{2}{*}{.000} & \multirow[t]{2}{*}{2.048} & \multirow[t]{2}{*}{870} & \multirow[t]{2}{*}{.041} \\
\hline & Mujer & 463 & 18.37 & 4.86 & & & & & \\
\hline
\end{tabular}

De igual manera, se observan diferencias estadísticamente significativas en la SWL según la edad, donde los estudiantes que tienen 18 años evidencian mayor satisfacción con la vida que los estudiantes de 15, 16 y 17 años (Tabla 3). Sin embargo, el valor de $\omega^{2}=0.01$ indicaría la ausencia de TE. Así pues, se podría afirmar que si bien al alcanzar la mayoría de edad se realiza una evaluación más positiva de la vida, estos resultados no permitirían una interpretación de su importancia práctica.

\section{Tabla 3.}

Satisfacción con la Vida según edad.

\begin{tabular}{lllllll}
\hline & & Suma de cuadrados & gl & Media cuadrática & F & p \\
\hline Satisfacción & Entre grupos & 235.338 & 4 & 58.834 & 2.911 & .021 \\
con la Vida & Dentro de grupos & 17524.959 & 867 & 20.213 & & \\
(SWLS) & Dotal & 17760.297 & 871 & & & \\
& Total & & & & \\
\hline
\end{tabular}

Finalmente, la satisfacción con la vida según institución educativa no manifiesta diferencias estadísticamente significativas ni importancia práctica $\left(\omega^{2}=0.00\right)$, es decir, el pertenecer a instituciones de varones, de mujeres o mixtas, no muestra diferencias en la evaluación que hacen los alumnos de su vida en términos generales (Tabla 4). 


\section{Tabla 4.}

Satisfacción con la Vida según institución educativa.

\begin{tabular}{lllllll}
\hline & & Suma de cuadrados & gl & Media cuadrática & F & p \\
\hline $\begin{array}{lllllll}\text { Satisfacción } \\
\text { con la Vida }\end{array}$ & Entre grupos & 118.399 & 4 & 29.600 & 1.455 & .214 \\
(SWLS) & Dentro de grupos & 17641.898 & 867 & 20.348 & & \\
& Total & 17760.297 & 871 & & & \\
\hline
\end{tabular}

\section{Discusión}

El presente estudio como objetivos, analizar las propiedades psicométricas de la SWLS de Diener et al. (1985) y comparar las puntuaciones de SWL en función del sexo, la edad y la institución de procedencia en un grupo de escolares de la ciudad de Arequipa.

Respecto al primer objetivo, los resultados del AFC proporcionan apoyo empírico para el modelo unidimensional de la SWLS. Asimismo, las cargas factoriales de cada ítem son todas significativas, aunque solo 4 ítems superan el valor de .70 que representan más de la mitad de la varianza explicada en cada ítem. Este resultado significa que la varianza del error para cada ítem es menor que la varianza explicada por la SWLS, lo que proporciona apoyo para la validez de constructo del SWLS (Hultell \& Gustavsson, 2008). La diferencia entre las cargas factoriales del ítem 1 y los cuatro restantes, evidencia que el primer ítem influye de forma diferente en la definición del constructo SWL. Así, para la muestra de escolares arequipeños, una adecuada interpretación de la puntuación de la SWLS está asociada con una ponderación diferente de cada ítem (Merino-Soto \& Salas, 2017).

Asimismo, los valores de los coeficientes de confiabilidad para variables latentes $(\omega=.99$ y $\mathrm{H}=.99)$ supone un adecuado nivel de consistencia interna del modelo unidimensional de la SWFLS. Cabe señalar que nuestros valores de confiabilidad, son superiores a los reportados por Alarcón (2000) para estudiantes universitarios de Lima, posiblemente, porque se utilizó un método 
diferente de cálculo. Mientras Alarcón utilizó el método de mitades partidas, en este estudio se calcularon los coeficientes $\omega$ y $\mathrm{H}$ que son útiles para modelos factoriales. Otro factor para la existencia de diferencias tiene que ver con la muestra, ya que en el estudio de Alarcón se trabajó con estudiantes de una universidad privada, y aquí se tuvo una muestra conformada por estudiantes de escuelas públicas de nivel secundario. Nuestros datos sugieren que la SWLS permite interpretaciones válidas y confiables para su uso en estudiantes arequipeños de colegios públicos con una edad de entre 14 y 18 años.

En relación al segundo objetivo, se reportó que los estudiantes varones tienen puntuaciones más altas en satisfacción con la vida, en comparación con las mujeres, aunque estas diferencias no presentan importancia práctica. Estos datos son similares a lo reportado en la literatura internacional donde no se presentan diferencias importantes (Casas et al. 2015; Froh et al., 2009; Gilman \& Huebner, 2006; Huebner et al., 2000; Huebner et al., 2004), pero difieren de los que indican que las mujeres tienen mayor SWL que los estudiantes varones (Laca, Verdugo \& Guzmán, 2005) o de aquellos estudios donde los hombres presentan mayores niveles de SWL (Bernal et al. 2015; Goldbeck et al., 2007; Moksnes \& Espnes, 2013; Neto, 1993; Suldo et al., 2015; Tarazona, 2005; Verkuyten, 1986). Mientras que en el Perú no se han reportado diferencias significativas de la SWL en función del sexo de los estudiantes involucrados (Alarcón, 2001; Salgado, 2009).

La edad, también mostró diferencias estadísticamente significativas de la SWL a favor de los estudiantes que tienen mayor edad, concretamente, quienes tienen más de 18 años, aunque hay una ausencia de importancia práctica. Aun así, las ligeras diferencias posiblemente se expliquen, por el hecho de que la mayoría de edad, implica para un estudiante, tener acceso a ciertas fuentes de diversión, que un menor de edad no tiene, como ingresar a discotecas, consumir bebidas alcohólicas, etc. No podemos empero, afirmar que esta sea la razón, porque no se han evaluado las fuentes de felicidad de los escolares (Alarcón, 2002), sin embargo, los estudios previos en Perú, 
con muestras de escolares y universitarios limeños, no registran diferencias significativas en función de la edad (Alarcón, 2001; Salgado, 2009). Otros estudios empero, con muestras con un amplio espectro etario, han reportado que conforme aumenta la edad, se obtienen puntajes más altos en la SWL (Baird, Lucas \& Donnellan, 2010). En ese sentido, Martínez (2004), también encontró que, en 570 personas de entre 16 y 65 años de la ciudad de Lima, quienes tienen mayor edad, tienen puntajes más altos en SWL.

Finalmente, con respecto la institución educativa de procedencia, no hemos encontrado diferencias significativas ni importancia práctica entre colegios mixtos o diferenciados, lo que corrobora algunos estudios locales que señalan que la educación mixta y diferenciada no representa diferencias significativas en la ocurrencia de conductas disruptivas o los niveles de autoestima de los escolares arequipeños (Gordillo, Cahuana \& Rivera, 2016). En el trabajo de Tarazona (2005), empero, se encontraron diferencias significativas en función del tipo de gestión de la escuela, a favor de las instituciones educativas de gestión privada de Lima.

Con este breve, pero original estudio, pues es el primero en abordar la SWL en la ciudad de Arequipa, se pone a disposición de los investigadores locales, un instrumento de medición, que, aunque es muy conocido en todo el mundo, no había sido validado en el Perú para escolares de educación secundaria. Con estos resultados se podrán realizar nuevas investigaciones psicométricas, correlacionales o experimentales, que nos permitan profundizar en la comprensión del bienestar subjetivo de las generaciones más jóvenes. Asimismo, nuestros resultados, dan cuenta del comportamiento de esta variable de estudio en función del sexo y la edad, que supone, además, diseñar estrategias para mejorar el nivel de satisfacción de los escolares arequipeños.

El estudio presenta varias limitaciones. Primero los participantes fueron solo estudiantes de cuarto y quinto grado de secundaria. Por lo tanto, hay una necesidad de examinar propiedades psicométricas para adolescentes en un amplio rango de edades. En segundo lugar, la evaluación de la validez 
discriminante no se incluyó en el presente estudio, y por lo tanto, se sugiere que se realice un estudio que utilice escalas que evalúen constructos diferentes a la SWL (por ejemplo, síntomas depresivos). Una tercera limitación, radica en que los hallazgos se basaron en autoinformes, estando por lo tanto, sujeto a un posible sesgo característico de estos procedimientos. Asimismo, se hace necesario una mayor información acerca de la naturaleza y direccionalidad de la relación entre la SWL y las variables sociodemográficas estudiadas más allá del corte transversal, diseño que se utilizó en el presente estudio. Así, futuras investigaciones deberían utilizar un diseño longitudinal que permitiría fortalecer el estudio al permitir que los cambios sean evaluados y comparados con el tiempo.

En conclusión, el estudio sugiere que la SWLS es un instrumento adecuado para evaluar la SWL en escolares arequipeños que es un importante predictor de la salud, el bienestar y el éxito, tanto académico como profesional (Diener, 2000). La SWLS resultaría beneficiosa en futuros estudios en niños de todo el rango de edad; además es recomendable su uso en estudios epidemiológicos y de investigación clínica. En ese sentido, este trabajo abre nuevas posibilidades de investigación sobre la variable SWL en la ciudad de Arequipa, pues no existen estudios previos sobre dicho constructo en nuestra ciudad. Con la SWLS pueden realizarse nuevas investigaciones y diseñarse estrategias de intervención, que permitan valorar a través de la satisfacción con la vida, otros constructos relacionados o su variabilidad en función de la aplicación de sesiones, programas o talleres educativos.

\section{Referencias}

Adler, A. (2017). Educación positiva: Educando para el éxito académico y para la vida plena. Papeles del Psicólogo, 38(1), 50-57. doi: https://doi. org/10.23923/pap.psicol2017.2821

Alarcón, R. (2000). Variables psicológicas asociadas con la felicidad. Persona, 3, 147-157. 
Alarcón, R. (2001). Relaciones entre felicidad, género, edad y estado conyugal. Revista de Psicología, 19(1), 27-46.

Alarcón, R. (2002). Fuentes de felicidad: ¿Qué hace a la gente feliz? Revista de Psicología 20(2), 169-196.

Alfaro, J., Guzmán, J., Sirlopú, D., García, C., Reyes, F., \& Gaudlitz, L. (2016). Propiedades psicométricas de la Escala de Satisfacción con la Vida en los Estudiantes (SLSS) de Huebner en niños y niñas de 10 a 12 años de Chile. Anales de Psicología, 32(2), 383-392. doi: https://doi. org/10.6018/analesps.32.2.217441

Arias, W. L., Espiñeira, E. \& Huamani, J. C. (2017). Representación de la Psicología Positiva en el Perú: un estudio bibliométrico en diez revistas científicas peruanas del 2000 al 2016. Artículo de investigación sometido a revisión.

Arrindell, W. A., Meeuwesen, L., \& Huyse, F. J. (1991). The Satisfaction With Life Scale (SWLS) - Psychometric properties in a nonpsychiatric medical outpatients sample. Personality and Individual Differences, 12(2), 117-123. doi: https://doi.org/10.1016/0191-8869(91)90094-R

Atienza, F. L., Pons, D., Balaguer, I., \& García-Merita, M. (2000). Propiedades psicométricas de la Escala de Satisfacción con la Vida en adolescentes. Psicothema, 12(2), 314-319.

Atienza, F. L., Balaguer, I., \& García-Merita, M. L. (2003). Satisfaction with life scale: Analysis of factorial invariance across sexes. Personality and Individual Differences, 35(6), 1255-1260. doi: https://doi.org/10.1016/ S0191-8869(02)00332-X

Ato, M., López, J. J., \& Benavente, A. (2013). Un sistema de clasificación de los diseños de investigación en psicología. Anales de Psicología, 29(3), 1038-1059. doi: https://doi.org/10.6018/analesps.29.3.178511

Baird, B. M., Lucas, R. E., \& Donnellan, M. B. (2010). Life satisfaction across the lifespan: Findings from two nationally representative panel studies. Social Indicators Research, 99(2), 183-203. doi: https://doi. org/10.1007/s11205-010-9584-9

Bernal, T., Daza, C., \& Jaramillo, P. (2015). Satisfacción con la vida y resiliencia en jóvenes en extraedad escolar. Revista Iberoamericana de Psicología, 8(2), 43-53. 
Bisquerra, R., \& Hernández, S. (2017). Psicología positiva, educación emocional y el programa aulas felices. Papeles del Psicólogo, 38(1), 5865. doi: https://doi.org/10.23923/pap.psicol2017.2822

Bollen, K. A. (1998). Structural equation models. En P. Armitage, T. Colton (Ed.). Encyclopedia of Biostatistics (pp. 4363-4372). Sussex, UK: Wiley.

Cabañero, M.J., Martínez, M.R., Cabrero, J., Orts, M.I., Reig, A., \& Tosal, B. (2004). Fiabilidad y validez de la Escala de Satisfacción con la Vida de Diener en una muestra de mujeres embarazadas y puérperas. Psicothema, 16(3), 448-455.

Çakar, F. F., Tagay, Ö. O., \& Karataş, Z. Z. (2015). Adolescents' life satisfaction: risky behaviors and hopelessness. International Journal on New Trends in Education \& Their Implications, 6(1), 55-62.

Carmines, E. G., \& McIver, J. P. (1981). Analyzing models with unobservable variables. En G. Bohrnstedt y E. Borgatta (Eds.) Social measurement: Current issues. (pp. 65-115). Beverly Hills, CA Sage.

Casas, F., Alfaro, J., Sarriera, J. C., Bedin, L., Grigoras, B., Bălțătescu, S., Malo, S., \& Sirlopú, D. (2015). El bienestar subjetivo en la infancia: estudio de la comparabilidad de 3 escalas psicométricas en 4 países de habla latina. Psicoperspectivas, 14(1), 6-18.

Casas, F., Figuer, C., Gonzalez, M., Malo, S., Alsinet, C., \& Subarroca, S. (2007). The well-being of 12- to 16-year old adolescents and their parents: Results from 1999 to 2003 Spanish samples. Social Indicators Research, 83(1), 87-115. doi: https://doi.org/10.1007/s11205-006-90591

Castro, A. (2012). La psicología positiva en América Latina. Desarrollos y perspectivas. Psciencia. Revista Latinoamericana de Ciencia psicológica, 4(2), 108-116.

Caycho-Rodríguez, T. (2017). Comparaciones entre más de dos grupos: aportes para el cálculo del tamaño del efecto. Investigación en Educación Médica. Avance online. Disponible en: http://dx.doi.org/10.1016/j. riem.2017.04.003

Chui, W. H., \& Wong, M. Y. (2016). Gender differences in happiness and life satisfaction among adolescents in Hong Kong: Relationships and self- 
concept. Social Indicators Research, 125(3), 1035-1051. doi: https://doi. org/10.1007/s11205-015-0867-z

Cohen, J. (1998). Statistical Power Analysis for the Behavioral Sciences. Hillsdale: Erlbaum.

Díaz, J. F., \& Martínez, P. (2004). Metas y satisfacción vital en adolescentes españoles y peruanos según sexo y nivel socioeconómico. Revista de Psicología, 22(1), 121-149.

Diener, E. (2000). Subjective well-being: The science of happiness and a proposal for a national index. American Psychologist, 55, 34-43. doi: https://doi.org/10.1037/0003-066X.55.1.34

Diener, E., Emmons, R. A., Larsen, R. J., \& Griffin, S. (1985). The Satisfaction with Life Scale. Journal of Personality Assessment, 49(1), 71-75. doi: https://doi.org/10.1207/s15327752jpa4901_13

Diener, E., Oishi, S., \& Lucas, R. (2003). Personality, culture, and subjective well-being: emotional and cognitive evaluations of life. Annual Review of Psychology, 54, 403-425. doi: https://doi.org/10.1146/annurev. psych.54.101601.145056

Diener, E., \& Ryan, K. (2009). Subjective well-being: A general overview. South African Journal of Psychology, 39(4), 391-406. doi: https://doi. org/10.1177/008124630903900402

Durak, M., Senol-Durak, E., \& Gencoz, T. (2010). Psychometric properties of the satisfaction with life scale among Turkish university students, correctional officers, and elderly adults. Social Indicators Research, 99(3), 413-429. doi: https://doi.org/10.1007/s11205-010-9589-4

Erdogan, B., Bauer, T. N., Truxillo, D. M., \& Mansfield, L. R. (2012). Whistle while you work: A review of the life satisfaction literature. Journal of Management, 38(4), 1038-1083. doi: https://doi. org/10.1177/0149206311429379

Ferguson, C. J. (2009). An effect size primer: A guide for clinicians and researchers. Professional Psychology: Research and Practice, 40(5), 532-538. doi: https://doi.org/10.1037/a0015808

Fouquereau, E., \& Rioux, L. (2002). Elaboration de l'Echelle de satisfaction de vie professionnelle (ESVP) en langue francaise: Une demarche 
exploratoire [Development of the French-language Professional Life Satisfaction Scale: An exploratory study]. Canadian Journal of Behavioural Science, 34(3), 210-215. doi: https://doi.org/10.1037/ h0087173

Frasquilho, D., Matos, M., Neville, F., Gaspar, T., \& Almeida, J. (2016). Parental Unemployment and Youth Life Satisfaction: The Moderating Roles of Satisfaction with Family Life. Journal of Child \& Family Studies, 25(11), 3214-3219. doi: https://doi.org/10.1007/s10826-0160480-z

Fritz, C. O., Morris, P. E., \& Richler, J. J. (2012). Effect size estimates: Current use, calculations, and interpretation. Journal of Experimental Psychology: General, 141(1), 2-18. doi: https://doi.org/10.1037/ a0024338

Froh, J. J., Yurkewicz, C., \& Kashdan, T. B. (2009). Gratitude and subjective well-being in early adolescence: Examining gender differences. Journal of Adolescence, 32(3), 633-650. doi: https://doi.org/10.1016/j. adolescence.2008.06.006

Fornell, C., \& Larcker, D.F. (1981). Evaluating structural equation models with unobservable variables and measurement error. Journal of Marketing Research, 18(1), 39-50. doi: https://doi.org/10.2307/3151312

Gilman, R., \& Huebner, E. S. (2001). Review of life satisfaction measures for adolescents. Behaviour Change, 17(3), 178-195. doi: https://doi. org $/ 10.1375 /$ bech.17.3.178

Gilman, R., \& Huebner, S. (2003). A review of life satisfaction research with children and adolescents. School Psychology Quarterly, 18(2), 192-205. doi: https://doi.org/10.1521/scpq.18.2.192.21858

Gilman, R., \& Huebner, E. S. (2006). Characteristics of adolescents who report very high life satisfaction. Journal of Youth and Adolescence, 35(3), 293-301. doi: https://doi.org/10.1007/s10964-006-9036-7

Goldbeck, L., Schmitz, T. G., Besier, T., Herschbach, P., \& Henrich, G. (2007). Life satisfaction decreases during adolescence. Quality of Life Research, 16(6), 969-979. doi: https://doi.org/10.1007/s11136-0079205-5 
Gordillo, E., Cahuana, M., \& Rivera, R. (2016). Conductas disruptivas y autoestima en escuelas mixtas y diferenciadas de Arequipa. Arequipa: Universidad Católica San Pablo.

Gouveia, V. V., Milfont, T. L., Da Fonseca, P. N., \& de Miranda Coelho, J. A. P. (2009). Life satisfaction in Brazil: Testing the psychometric properties of the satisfaction with life scale (SWLS) in five Brazilian samples. Social Indicators Research, 90(2), 267-277. doi: https://doi.org/10.1007/ s11205-008-9257-0

Greenspoon, P. J., \& Saklofske, D. H. (2001). Toward an integration of subjective well-being and psychopathology. Social Indicators Research, 54(1), 81-108. doi: https://doi.org/10.1023/A:1007219227883

Hair, J., Anderson, R., Tatham, R., \& Black, W. (2014). Análisis Multivariante. México, D. F.: Prentice Hall.

Hancock, G. R., \& Mueller, R. O. (2001). Rethinking construct reliability within latent variable systems. En R. Cudeck, S. H. C. du Toit \& D. Sörbom (Eds.), Structural equation modeling: Past and present. A Festschrift in honor of Karl G. Jöreskog (pp. 195-261). Chicago: Scientific Software International.

Hernández, R., Fernández, C. \& Baptista, P. (2010). Metodología de la investigación. México: McGraw-Hill.

Hu, L.T., \& Bentler, P.M. (1999). Cutoff criteria for fit indexes in covariance structure analysis: Conventional criteria versus new alternatives. Structural Equation Modeling: A Multidisciplinary Journal, 6(1), 1-55. doi: https://doi.org/10.1080/10705519909540118

Huamani, J. C., \& Ccori, J. (2016). Respuesta al sentido de vida en adolescentes. Revista de Psicología de Arequipa, 6(1), 331-348.

Huebner, E. S., Drane, W., \& Valois, R. F. (2000). Levels and demographic correlates of adolescent life satisfaction reports. School Psychology International, 21(3), 281-292. doi: https://oi. org/10.1177/0143034300213005

Huebner, E. S., Suldo, S., Valois, R. F., Drane, J. W., \& Zullig, K. (2004). Brief multidimensional students' life satisfaction scale: sex, race, and grade effects for a high school sample. Psychological Reports, 94(1), 351-356. doi: https://doi.org/10.2466/pr0.94.1.351-356 
Hultell, D., \& Gustavsson, J. P. (2008). A psychometric evaluation of the satisfaction with life scale in a Swedish nationwide sample of university students. Personality and Individual Differences, 44(5), 1070-1079. doi: https://doi.org/10.1016/j.paid.2007.10.030

Johnson, B., \& Stevens, J. J. (2001). Exploratory and confirmatory factor analysis of the School Level Environment Questionnaire (SLEQ). Learning Environments Research, 4(3), 325-344. doi: https://doi. org/10.1023/A:1014486821714

Laca, F. A., Verdugo, J. C., \& Guzmán, J. (2005). Satisfacción con la vida de algunos colectivos mexicanos: una discusión sobre la psicología del bienestar subjetivo. Enseñanza e Investigación en Psicología, 10(2), 325-336.

Laranjeira, C. A. (2009). Preliminary validation study of the Portuguese version of the satisfaction with life scale. Psychology, Health \& Medicine, 14(2), 220-226. doi: https://doi.org/10.1080/13548500802459900

Lent, R. W., do Céu Taveira, M., Sheu, H. B., \& Singley, D. (2009). Social cognitive predictors of academic adjustment and life satisfaction in Portuguese college students: A longitudinal analysis. Journal of Vocational Behavior, 74(2), 190-198. doi: https://oi.org/10.1016/j. jvb.2008.12.006

Lewis, A. D., Huebner, E. S., Malone, P. S., \& Valois, R. F. (2011). Life satisfaction and student engagement in adolescents. Journal of Youth and Adolescence, 40(3), 249-262. doi: https://doi.org/10.1007/s10964010-9517-6

Lewis, C. A., Shevlin, M. E., Smekal, V., \& Dorahy, M. J. (1999). Factor structure and reliability of a Czech translation of the Satisfaction With Life Scale among Czech university students. Studia Psychologica, 41(3), 239-244

Lim, Y. J. (2015). Psychometric characteristics of the Korean version of the Satisfaction with Life Scale adapted for children. Canadian Journal of School Psychology, 30(3), 246-251. doi: https://doi. org/10.1177/0829573515590012 
Luna, A. C., Laca, F. A., \& Mejía, J. C. (2011). Bienestar subjetivo y satisfacción con la vida de familia en adolescentes mexicanos de bachillerato. Psicología Iberoamericana, 19(2), 17-26.

Martínez, P. (2004). Perspectiva temporal futura y satisfacción con la vida a lo largo del ciclo vital. Revista de Psicología, 22(2), 215-252.

Martínez, P. (2006). Perspectiva futura del Perú y el nivel de satisfacción con él. Liberabit, 12, 113-121.

Martínez-Antón, M., Buelga, S., \& Cava, M. J. (2007). La satisfacción con la vida en la adolescencia y su relación con la autoestima y el ajuste escolar. Anuario de Psicología, 38(2), 293-303.

McDonald, R. P. (1999). Test theory: A unified treatment. Mahwah, NJ: Erlbaum.

Merchán, I. M., Bermejo, M. L., \& González, J. D. (2014). Eficacia de un programa de educación emocional en educación primaria. Revista de Estudios e Investigación en Psicología y Educación, 1(1), 91-99.

Merino-Soto, C., \& Salas, E. (2017). Escala breve de búsqueda de sensaciones (BSSS): estructura latente de las versiones de 8 y 4 ítems en adolescentes peruanos. Adicciones. doi: 10.20882/adicciones. 842

Meuleners, L. B., Lee, A. H., Binns, C. W., \& Lower, A. (2003). Quality of life for adolescents: assessing measurement properties using structural equation modelling. Quality of Life Research, 12(3), 283-290. doi: https://doi.org/10.1023/A:1023221913292

Moksnes, U. K., \& Espnes, G. A. (2013). Self-esteem and life satisfaction in adolescents-gender and age as potential moderators. Quality of Life Research, 22(10), 2921-2928. doi: https://doi.org/10.1007/s11136-0130427-4

Moksnes, U. K., Løhre, A., Byrne, D. G., \& Haugan, G. (2014). Satisfaction with life scale in adolescents: Evaluation of factor structure and gender invariance in a Norwegian sample. Social Indicators Research, 118(2), 657-671. doi: https://doi.org/10.1007/s11205-013-0451-3

Montero, I., \& León, O. G. (2007). A guide for naming research studies in Psychology. International Journal of Clinical and Health Psychology, 7(3), 847-862. 
Neto, F. (1993). The satisfaction with life scale: Psychometrics properties in an adolescent sample. Journal of Youth and Adolesence, 22(2), 125-134. doi: https://doi.org/10.1007/BF01536648

Neto, F. (2001). Satisfaction with life among adolescents from immigrant families in Portugal. Journal of Youth and Adolescence, 30(1), 53-67. doi: https://doi.org/10.1023/A:1005272805052

Oishi, S. (2006). The concept of life satisfaction across cultures: An IRT analysis. Journal of Research in Personality, 40(4), 411-423. doi: https:// doi.org/10.1016/j.jrp.2005.02.002

Oladipo, S. E., \& Balogun, S. K. (2012). How suitable is the satisfaction with Life Scale for use on adolescents in Nigeria. Science Journal of Psychology, 2012(2), 1-3. Recuperado de: http://www.sjpub.org/sjpsych/ sjpsych-228.pdf

Oros, L. B., Manucci, V., \& Richaud, M. C. (2011). Desarrollo de emociones positivas en la niñez. Lineamientos para la intervención escolar. Educación y Educadores, 14(3), 493-509.

Palomera, R. (2017). Psicología positiva en la escuela: un cambio con raíces profundas. Papeles del Psicólogo, 38(1), 66-71. doi: https://doi. org/10.23923/pap.psicol2017.2823

Pavot, W., Diener, E. D., Colvin, C.R., \& Sandvik, E.(1991). Further validation of the Satisfaction with Life Scale: Evidence for the cross-method convergence of well-being measures. Journal of Personality Assessment, 57(1), 149-161. doi: https://doi.org/10.1207/s15327752jpa5701_17

Pavot, W., \& Diener, E. (1993). Review of the satisfaction with life scale. Psychological Assessment, 5(2), 164-172. doi: https://doi. org/10.1037/1040-3590.5.2.164

Pavot, W., \& Diener, E. (2008). The satisfaction with life scale and the emerging construct of life satisfaction. The Journal of Positive Psychology, 3(2), 137-152. doi: https://doi.org/10.1080/17439760701756946

Pons, D., Atienza, F. L., Balaguer, I., \& Garc1'a-Merita, M. L. (2002). Propiedades psicometricas de la Escala de Satisfacción con la Vida en personas de tercera edad. Revista Iberoamericana de Diagnóstico y Evaluación Psicológica, 13, 71-82. 
Povedano, A., Hendry, L. B., Ramos, M. J., \& Varela, R. (2011). Victimización escolar: clima familiar, autoestima y satisfacción con la vida desde una perspectiva de género. Psychosocial Intervention, 20(1), 5-12. doi: https://doi.org/10.5093/in2011v20n1a1

Proctor, C. L., Linley, P. A., \& Maltby, J. (2009a). Youth life satisfaction measures: A review. The Journal of Positive Psychology, 4(2), 128-144. doi: https://doi.org/10.1080/17439760802650816

Proctor, C. L., Linley, P. A., \& Maltby, J. (2009b). Youth life satisfaction: A review of the literature. Journal of Happiness Studies, 10(5), 583-630. doi: https://doi.org/10.1007/s10902-008-9110-9

Proctor, C., Linley, P. A., \& Maltby, J. (2010). Very happy youths: Benefits of very high life satisfaction among adolescents. Social Indicators Research, 98(3), 519-532. doi: https://doi.org/10.1007/s11205-0099562-2

Raykov, T., \& Hancock, G. R. (2005). Examining change in maximal reliability for multiple-component measuring instruments. British Journal of Mathematical and Statistical Psychology, 58(1), 65-82. doi: https://doi.org/10.1348/000711005X38753

Rivera, R., Arias, W. L., \& Cahuana, M. (2017). Perfil familiar de adolescentes con sintomatología depresiva en la ciudad de Arequipa, Perú. Revista Chilena de Neuropsiquiatría. (artículo sometido a revisión).

Rodgers, V., Neville, S., \& La Grow, S. (2017). Health, functional ability and life satisfaction among older people 65 years and over: a cross-sectional study. Contemporary Nurse, 53(3), 1-9. doi: https://doi.org/10.1080/103 76178.2017.1319286

Sachs, J. (2004). Validation of the satisfaction with life scale in a sample of Hong Kong university students. Psychologia, 46(4), 225-234. doi: https://doi.org/10.2117/psysoc.2003.225

Salgado, A. C. (2006). Felicidad en estudiantes de universidades nacionales de Perú, Bolivia, Paraguay y Argentina. Cultura, 20(1), 531-552.

Salgado, A. C. (2009). Felicidad, resiliencia y optimismo en estudiantes de colegios nacionales de la ciudad de Lima. Liberabit, 15(2), 133-141. 
Sancho, P., Galiana, L., Gutierrez, M., Francisco, E. H., \& Tomás, J. M. (2014). Validating the Portuguese version of the satisfaction with life scale in an elderly sample. Social Indicators Research, 115(1), 457-466. doi: https://doi.org/10.1007/s11205-012-9994-y

Silva, A. D., do Céu Taveira, M., Marques, C., \& Gouveia, V. V. (2015). Satisfaction with life scale among adolescents and young adults in Portugal: Extending evidence of construct validity. Social Indicators Research, 120(1), 309-318. doi: https://doi.org/10.1007/s11205-0140587-9

Simões, A. (1992). Ulterior validação de uma escala de satisfação com a vida (SWLS). Revista Portuguesa de Pedagogia, 26(3), 503-515.

Suldo, S. M., \& Shaffer, E. J. (2008). Looking beyond psychopathology: The dual-factor model of mental health in youth. School Psychology Review, 37(1), 52-68.

Suldo, S. M., Huebner, E. S., Friedrich, A. A., \& Gilman, R. (2009). Life satisfaction. In R. Gilman, E. S. Huebner, \& M. Furlong (Eds.), Handbook of positive psychology in the schools (pp. 27-35). New York: Routledge.

Suldo, S. M., \& Shaunessy-Dedrick, E. (2013). The psychosocial functioning of high school students in academically rigorous programs. Psychology in the Schools, 50(8), 823-843. doi: https://doi.org/10.1002/pits.21708

Suldo, S. M., Minch, D. R., \& Hearon, B. V. (2015). Adolescent life satisfaction and personality characteristics: Investigating relationships using a five factor model. Journal of Happiness Studies, 16(4), 965-983. doi: https://doi.org/10.1007/s10902-014-9544-1

Tan, J., Yang, W., Ma, H., \& Yu, Y. (2016). Adolescents' core self-evaluations as mediators of the effect of mindfulness on life satisfaction. Social Behavior and Personality: an International Journal, 44(7), 1115-1122. doi: https://doi.org/10.2224/sbp.2016.44.7.1115

Tarazona, D. (2005). Autoestima, satisfacción con la vida y condiciones de habitabilidad en adolescentes estudiantes de quinto año de media. Un estudio factorial según pobreza y sexo. Revista de Investigación en Psicología, 8(2), 57-65.

Totan, T., Özer, A., \& Özmen, O. (2017). The role of hope, life satisfaction, and motivation in bullying among adolescents. International Online 
Journal of Educational Sciences, 9(2), 391-400. doi: https://doi. org/10.15345/iojes.2017.02.008

Vassar, M. (2008). A note on the score reliability for the Satisfaction With Life Scale: An RG study. Social Indicators Research, 86(1), 47-57. doi: https://doi.org/10.1007/s11205-007-9113-7

Velásquez, C., Dioses, A., Chávez, J., Pomalaya, R., Cavero, V., Cabrera, S., Velásquez, N., \& Egúsquiza, B. (2016). Personalidad y satisfacción por áreas vitales en estudiantes de psicología de una universidad pública de Lima. Revista de Investigación en Psicología, 19(1), 81-97.

Ventura-León, J.L. \& Caycho-Rodríguez, T. (2017). El coeficiente omega: un método alternativo para la estimación de la confiabilidad. Revista Latinoamericana de Ciencias Sociales, Niñez y Juventud. 15(1), 625627.

Verkuyten, M. (1986). The impact of ethnic and sex differences on happiness among adolescents in the Netherlands. The Journal of Social Psychology, 126(2), 259-260.

Vittersø, J., Biswas-Diener, R., \& Diener, E. (2005). The divergent meanings of life satisfaction: Item response modeling of the Satisfaction With Life Scale in Greenland and Norway. Social Indicators Research, 74(2), 327348. doi: https://doi.org/10.1007/s11205-004-4644-7

Wixom, B. H., \& Todd, P. A. (2005). A theoretical integration of user satisfaction and technology acceptance. Information Systems Research, 16(1), 85-102.

Woynarowska, B., Tabak, I., \& Mazur, J. (2004). Self-reported health and life satisfaction in school-aged children in Poland and other countries in 2002. Medycyna Wieku Rozwojowego, 8(3), 535-550.

$\mathrm{Wu}, \mathrm{C}$., \& Yao, G. (2006). Analysis of factorial invariance across gender in the Taiwan version of the satisfaction with life scale. Personality and Individual Differences, 40(6), 1259-1268. doi: https://doi.org/10.1016/j. paid.2005.11.012 